\title{
Synthèse
}

\section{Exploitation agricole et agriculture durable}

\author{
Mohamed Gafsi \\ École nationale de formation agronomique, \\ Unité mixte de recherche (UMR) \\ "Dynamiques rurales", \\ BP 22687 , \\ 2, route de Narbonne, \\ 31326 Castanet Tolosan \\ $<$ mohamed.gafsi@educagri.fr>
}

\begin{abstract}
Résumé
Cet article propose des réflexions théoriques, avec l'appui d'un exemple empirique, pour une approche renouvelée de l'exploitation agricole adaptée aux exigences de la durabilité et de la multifonctionnalité de l'agriculture. Nous proposons, premièrement, une approche élargie des ressources stratégiques mobilisables par l'exploitation agricole, en incorporant en plus des capitaux classiques - physique, humain et financier - le capital naturel et le capital social. Ce cadre théorique s'appuie sur l'approche fondée sur les ressources. Deuxièmement, en partant de la théorie des parties prenantes, l'exploitation agricole est représentée en tant qu'organisation insérée dans des réseaux d'acteurs et dans un tissu d'influences multiples agissant sur les décisions stratégiques des agriculteurs. La théorie des parties prenantes permet d'apporter un éclairage sur les frontières de l'exploitation agricole et sur les rapports de l'exploitation agricole aux nouveaux acteurs non agricoles.
\end{abstract}

Mots clés : exploitation agricole ; agriculture durable ; théorie économique ; parties prenantes.

Thèmes : systèmes agraires ; économie et développement rural ; méthodes et outils.

\section{Abstract \\ Farms and sustainable agriculture}

This paper offers a theoretical analysis for a renewed approach to farming suitable to the requirements of sustainability and multifunctionality and provides an empirical example. We propose first a broader approach to strategic resources that can be mobilized by farms. These include not only the classic forms of capital - physical, human, and financial but also natural and social capital. This theoretical framework relies on a resource-based approach. Second, starting from stakeholder theory, the farm is represented as an organization inserted in stakeholder networks and a web of multiple influences acting on the farmers' strategic decisions. Stakeholder theory offers a new light on the farm's borders and on its relations with other non-agricultural participants and actors.

Key words: farms; sustainable agriculture; economic theories; stakeholders.

Subjects: farming systems; economy and rural development; tools and methods.

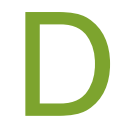
epuis la fin des années 1990, les perspectives de développement d'une agriculture durable et en phase avec les attentes nouvelles de la société sont au centre des débats de politiques agricoles (Landais, 1998). Les nouvelles orientations de politiques agricoles en termes de développement durable et d'écoconditionnalité correspondent à un changement important dans le contexte d'action des agriculteurs. Cela n'est pas sans conséquences sur la façon d'aborder l'exploitation agricole. Le développement d'une agriculture durable fait émerger des interrogations sur la nature de l'exploitation agricole, ses logiques de fonctionnement, ses frontières, etc.

Cet article vise à proposer des pistes de réflexion théorique dont l'objectif serait de jeter les bases d'une approche renouvelée de l'exploitation agricole dans le cadre du développement durable. Les réflexions théoriques proposées se réfèrent aux développements récents en sciences de gestion des courants de la théorie des ressources et de la théorie des parties prenantes. 
La théorie des ressources permet de prendre en compte les dimensions environnementale et sociale dans le modèle de l'exploitation agricole. La théorie des parties prenantes permet d'apporter un éclairage sur les frontières de l'exploitation agricole, sur les rapports de l'exploitation agricole aux nouveaux acteurs non agricoles et sur la logique de fonctionnement transversale (territoriale).

Dans un premier temps, nous analyserons comment la durabilité de l'agriculture suppose un nouveau regard sur l'approche de l'exploitation agricole. Après avoir cerné les enjeux de ce nouveau contexte d'action, nous discuterons dans un deuxième temps des pistes de réflexion pour proposer une approche de l'exploitation agricole répondant aux exigences de la durabilité. Enfin, dans un troisième temps, à partir de données empiriques, nous montrerons comment on peut mobiliser cette nouvelle approche de l'exploitation agricole. Les données analysées sont issues de 70 enquêtes réalisées auprès d'agriculteurs dans le pays Midi-Quercy à l'est du département du Tarn-et-Garonne.

\section{Agriculture durable : définition et implications sur l'approche de l'exploitation agricole}

Godard et Hubert (2002) proposent de définir l'agriculture durable par deux composantes essentielles, assumées simultanément :

- la viabilité d'un système d'exploitation ou la "durabilité autocentrée ", c'est-àdire sa capacité à continuer à produire dans le futur, en entretenant les ressources naturelles et le capital humain (formation, compétences, etc.) ;

- la contribution du système d'exploitation à la durabilité du territoire auquel il appartient. Cette seconde composante comprend donc l'insertion dans l'économie locale, l'offre des services de proximité, le maintien et la création de l'emploi et milieu rural, la production de services environnementaux, etc.

La seconde composante de l'agriculture durable, qui constitue indéniablement l'originalité de l'approche française de la durabilité ${ }^{1}$, est au cour de la multifonctionnalité de l'agriculture. Selon l'Organisation de coopération et de développement économiques (OCDE) (2005), "la multifonctionnalité correspond à l'idée que l'agriculture, outre la production d'aliments et de fibres, fournit toute une série de produits autres que les produits de base, tels que les aménités rurales et environnementales ou la sécurité des approvisionnements, et contribue à la pérennité des zones rurales. "On associe ainsi à l'agriculture, dans le cadre d'un nouveau contrat social (Landais, 1998), des objectifs multiples qui concernent non seulement sa fonction économique classique de production des produits alimentaires mais aussi ses fonctions environnementale et sociale.

La mise en ouvre d'une agriculture durable et multifonctionnelle, telle qu'elle est définie précédemment, implique une nouvelle approche de l'exploitation agricole. Cela pour deux raisons. Premièrement, la viabilité du système d'exploitation (ou la durabilité autocentrée) suppose une approche élargie des ressources mobilisées par l'agriculteur. En effet, on peut se poser la question des facteurs qui influencent, voire déterminent, les capacités de l'exploitation à continuer à produire dans le futur. Du point de vue économique, ces capacités de l'exploitation résident dans la valeur des flux de services obtenus de l'utilisation des actifs spécifiques qui sont sous le contrôle de l'agriculteur (Legg, 1999). Ces actifs comprennent non seulement les capitaux classiques qui rentrent dans le processus de production agricole (capitaux physiques, financiers et humains) mais aussi les ressources relatives aux capitaux naturel et social (Pretty et Hine, 2002). Comme pour les capitaux classiques, l'accumulation et la valorisation des capitaux naturel et social sont nécessaires à la viabilité de l'exploitation agricole. En étudiant les pratiques gestionnaires des agriculteurs (leur rationalité), Chia (1987) avait mis en évidence l'importance de capital social et du capital culturel, en plus du capital économique, dans les choix et les pratiques des agriculteurs. Il est important de souligner alors qu'avec des impacts négatifs de l'agriculture sur la disponibilité des ressources naturelles, sur la qualité environnementale et des

\footnotetext{
$\overline{1}$ Les approches anglo-saxonnes se limitent à
} la première composante de la durabilité. produits alimentaires, sur le bien-être et la vitalité du milieu rural, etc., la viabilité de l'exploitation agricole elle-même sera considérablement diminuée. Cette thèse suppose un nouveau regard sur l'exploitation agricole et les ressources qu'elle mobilise.

Deuxièmement, en lien avec la seconde composante de la durabilité (la contribution à la durabilité du territoire), l'agriculture durable fait référence à une importante dimension territoriale. En effet, les services environnementaux et sociaux fournis par l'agriculture renvoient à la dimension spatiale de cette dernière et à son rôle d'acteur social dans le territoire, bref au lien intrinsèque de l'agriculture à son territoire (Gafsi, 2003). Cette dimension territoriale redessine les rapports entre les agriculteurs et les autres acteurs non agricoles du monde rural ${ }^{2}$ (Rémy, 2001). Par conséquent, les agriculteurs ont en face d'eux de nouveaux acteurs avec de nouvelles attentes et des besoins en matière d'espace. Cela a des implications fortes sur les logiques de fonctionnement de l'exploitation (logique verticale de filière versus logique horizontale de territoire ?) et sur les frontières de l'exploitation et de l'action de l'agriculteur, ce qui pose de nombreuses interrogations théoriques sur la nature de l'exploitation, ses frontières et ses fonctions/responsabilités.

\section{Vers une approche renouvelée de l'exploitation agricole}

L'exploitation agricole est une notion complexe et multidimensionnelle. Selon les approches disciplinaires des chercheurs, elle est appréhendée comme une unité de production (Chombart de Lauwe et al., 1964), une cellule sociale et familiale (Barthez, 1982; Ait Abdelmalek, 2000), un centre de décision et/ou un

\footnotetext{
${ }^{2}$ A titre d'exemple, la loi d'orientation agricole (LOA) de 1999 a élargi la composition des Commissions départementales d'orientation de l'agriculture (CDOA) à de nouveaux acteurs: le commerce indépendant de l'alimentation, les associations de protection de l'environnement, I'artisanat, les associations de défense des consommateurs.
} 


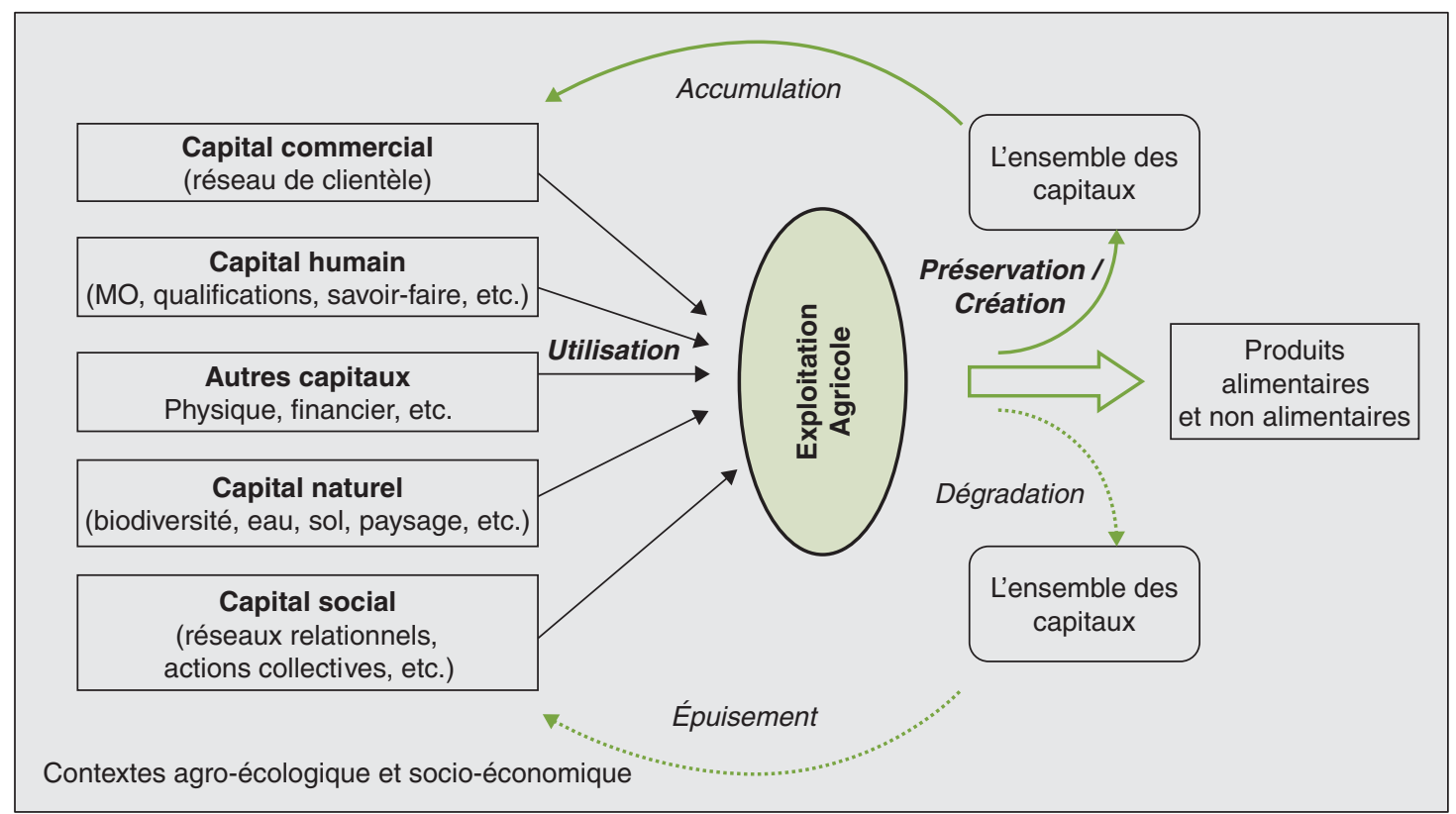

Figure 1. Approche basée sur les ressources d'une exploitation agricole, inspirée du modèle asset-based de Pretty et Hine (2002).

Figure 1. Resource-based view of a farm, inspired by the "asset-based" model developed by Pretty and Hine (2002).

système famille-exploitation (Brossier et al., 2003) ${ }^{3}$. Soulignons que ces différentes conceptions ne sont pas contradictoires. Bien au contraire, elles fondent la richesse des approches de l'exploitation agricole. D'où d'ailleurs le recours à une approche globale et une modélisation systémique de l'exploitation (Bonneviale et al., 1989 ; Marshall et al., 1994). Cela étant précisé, nous allons nous limiter désormais à la dimension économique, qui reste tout de même une dimension essentielle de l'exploitation agricole.

Du point de vue économique, l'exploitation peut être vue comme une unité de production agricole qui combine et utilise des facteurs de production pour produire des biens et services en vue de réaliser les objectifs fixés par l'agriculteur et sa famille. Dans l'approche microéconomique standard de l'exploitation agricole, référence est généralement faite aux seuls facteurs de production relevant des capitaux physiques (foncier, matériel, bâtiment), financiers et humains. Or les récents développements dans le domaine de la gestion d'entreprise montrent la nécessité d'élargir le cadre d'analyse des

\footnotetext{
${ }^{3}$ Voir aussi Laurent et al. (2003) pour une revue sélective des modèles de l'exploitation agricole en France. Pour les approches anglosaxonnes voir Gasson et Errington (1993), Gray (1998), Kay et Edwards (1999).
}

facteurs de production pour y intégrer d'autres types de capitaux. Il s'agit de l'approche basée sur les ressources (resource-based view) qui a connu son développement dans la dernière décennie (Wernerfelt, 1984 ; Wernerfelt, 1995 ; Barney, 1991). Cette approche considère qu'une entreprise fonde son développement sur des ressources stratégiques qui sont " difficilement imitables, difficilement substituables et difficilement échangeables " (Arregle, 1996). Ces ressources concernent non seulement les capitaux classiques (physique, financier, commercial et humain), mais aussi le capital naturel (Ekins et al., 2003; Russo, 2003) et le capital social (Chia, 1987; Coleman, 1988 ; Nahapiet et Ghoshal, 1998 ; Putnam, 2000).

Le capital naturel correspond au stock des biens (végétaux, animaux, minéraux, etc.) et services fournis (cycle d'absorption des déchets, par exemple) par les écosystèmes pour satisfaire les besoins humains. Ekins et al. (2003) proposent un cadre d'analyse du capital naturel, structuré par quatre éléments environnementaux : l'air, l'eau, le sol et les habitats.

Le capital social est "la somme des ressources effectives et potentielles intégrées dans, disponibles à travers ou découlant du réseau de relations que possède un individu ou une unité sociale " (Nahapiet et Ghoshal, 1998 ). Le capital social pré- sente principalement deux dimensions : l'une, structurelle, qui renvoie à la structure du réseau de relations entre les acteurs, et l'autre, relationnelle, correspondant au contenu des relations en termes de normes et de confiance qui résultent du réseau de relations. Deux types de liens sont identifiés comme importants dans les réseaux de relations: les liens forts dans le groupe d'acteurs (bonding, selon Putnam (2000)) et les liens faibles avec des acteurs hors du groupe (bridging).

En partant de l'approche fondée sur les ressources, l'exploitation agricole peut être alors modélisée en considérant les différents types de capitaux. De plus, dans une perspective de durabilité, l'exploitation agricole non seulement utilise les ressources relevant de ces capitaux, mais aussi contribue à la préservation et/ou à la création de ces ressources (figure 1). Il s'agit là d'un changement important dans la logique d'action des agriculteurs : d'une logique centrée uniquement sur l'utilisation des ressources selon le schéma simple de maximisation à court terme de la fonction de production, à une logique fondée sur la dynamique récursive de valorisation, de préservation et de création de ressources. Toujours dans une perspective de durabilité, notre approche de l'exploitation agricole se rapproche du modèle de l'agriculture 
durable développé par Pretty et Hine (2002), selon lequel les systèmes durables sont ceux qui assurent le renouvellement et favorisent l'accumulation d'un stock de capitaux.

Soulignons les spécificités des deux capitaux - social et naturel - quant à leur processus d'utilisation et d'accumulation. En effet, ils ont une importante dimension collective, parce que leur accumulation et utilisation reposent sur des formes de coopération et de coordination des acteurs (Beletti et al., 2002 ; Hagedorn et al., 2002 ; Pretty, 2003). Les autres capitaux (physique, capital financier, commercial et humain) sont, quant à eux, propres à un individu et font l'objet principalement de stratégies individuelles.

La dimension collective des deux capitaux - naturel et social - fait que leur accumulation et leur utilisation dépassent les frontières classiques de l'exploitation. Elles font intervenir d'autres acteurs ruraux - agricoles ou non agricoles - qui sont concernés par ces capitaux. En effet, pour pouvoir bénéficier de ces ressources stratégiques relevant de ces deux capitaux, l'agriculteur doit prendre en compte les intérêts et les attentes des autres acteurs concernés. La théorie des parties prenantes représente un cadre d'analyse pertinent de cette situation (Freeman, 1984; Mitchell et al., 1997 ; Sharma, 2001 ; Jonker et al., 2002). Selon cette théorie, pour réaliser ses objectifs, un chef d'entreprise doit comprendre et répondre aux besoins et aspirations d'acteurs faisant partie de l'environnement de l'entreprise. Ces acteurs, dits "parties prenantes", sont "tout groupe qui peut affecter ou être affecté par la réalisation des objectifs de l'entreprise" (Freeman, 1984). Vue sous l'angle de cette théorie, l'exploitation agricole est une organisation insérée dans des dynamiques d'acteurs et un tissu d'influences multiples agissant sur les décisions stratégiques de l'agriculteur. Les parties prenantes ne sont pas seulement les acteurs traditionnels d'amont et d'aval, mais aussi d'autres acteurs ruraux, y compris les consommateurs. Par conséquent, en plus des impératifs économiques des filières de production, le fonctionnement des exploitations obéit aussi à des contraintes (/opportunités) locales au niveau du territoire. La prise en compte des attentes des acteurs non agricoles élargit le champ d'action, et par conséquent la responsabilité, de l'agriculteur au-delà des frontières traditionnelles de son exploitation.

\section{Exemple de l'ancrage territorial \\ des exploitations agricoles ${ }^{4}$}

Cette approche de l'exploitation agricole a guidé le programme de recherche sur l'ancrage territorial des exploitations agricoles, mené dans le pays Midi-Quercy, à l'est du département du Tarn et Garonne. Soixante-dix exploitations agricoles, tirées au hasard ${ }^{5}$, ont été enquêtées.

\section{L'ancrage territorial vu à travers l'approche basée sur les ressources}

En partant de l'approche basée sur les ressources, l'ancrage territorial est défini comme un " processus de valorisation, de préservation et de production des ressources spécifiques du territoire". Ces ressources relèvent non seulement du capital physique, humain et financier, mais également du capital naturel et social. La question maintenant est : comment peut-on apprécier le degré d'ancrage d'une exploitation? En d'autres termes, comment peut-on apprécier les cinq types de capitaux et mesurer leur importance au niveau des pratiques agricoles dans l'exploitation? Le tableau 1 présente la déclinaison de ces capitaux en indicateurs de pratiques agricoles pertinents et simples à renseigner.

La démarche méthodologique consiste à attribuer, par la suite, une note de 0 à 5 à chaque indicateur de pratique, selon la présence de cette pratique. La somme totale des points constitue un indicateur synthétique d'ancrage territorial de l'exploitation. Partant de cet indicateur, une analyse de données a été conduite sur les 70 exploitations. Une typologie des exploitations selon le degré et la nature d'ancrage a été produite. Elle a

\footnotetext{
$\overline{4}$ Les travaux sur l'ancrage territorial des exploitations ont été réalisés par des chercheurs de l'Unité mixte de recherche "Dynamiques rurales » (M. Gafsi, B. Legagneux, G. Nguyen), dans le cadre du projet de recherche financé par le "Programme de recherche Sur et Pour le Développement Régional" en MidiPyrénées animé par l'Inra de Toulouse. Pour plus de détails voir Gafsi et al. (2002) et Nguyen et al. (2004).

${ }^{5}$ À partir de bases de données constituées par la Chambre d'agriculture.
}

permis de distinguer cinq groupes : deux groupes, A et B, présentant un degré d'ancrage élevé (notes moyennes, respectivement 26 et 24,3) ; deux groupes, C et $\mathrm{E}$, ayant un degré d'ancrage moyen (notes moyennes, respectivement 19,7 et 17 ; et un groupe $\mathrm{D}$ de faible ancrage (note moyenne, 12,5). Nous nous contentons ici de présenter les deux premiers groupes qui ont un degré d'ancrage élevé ${ }^{6}$. Dans un premier cas (groupe A), le degré d'ancrage est en lien avec la nature du système de production: les exploitations préservent au mieux le capital naturel et ne cherchent pas à développer le niveau de production mais plutôt à le valoriser par la transformation des produits et par la vente directe. Dans un second cas (groupe B), le degré important d'ancrage territorial est acquis par le profil personnel de l'agriculteur, qui se traduit notamment par la valorisation du capital social et la mâtrise d'un savoir-faire spécifique.

\section{Dynamique d'ancrage et approche des parties prenantes}

L'analyse de l'historique des exploitations a permis d'identifier deux dynamiques principales d'ancrage chez les exploitations présentant les meilleures notes (groupes A et B): l'ancrage territorial comme le résultat d'un changement d'orientation de système de production, et l'ancrage par accumulation progressive de capitaux.

La première dynamique a été observée dans des exploitations ayant d'une part des systèmes de production diversifiés permettant plus aisément de préserver et de valoriser le capital naturel et, d'autre part, des pratiques de transformation et de vente directe des produits agricoles. Les agriculteurs possèdent, de par leur origine non agricole ou leur longue expérience de travail non agricole, une ouverture sur le monde non agricole et des pratiques d'innovation. Cette première dynamique d'ancrage est le fruit d'un choix stratégique de l'agriculteur à un moment donné dans le cycle de vie de l'exploitation, le plus souvent lors d'une nouvelle installation ou d'une réorientation de système de production.

La seconde dynamique d'ancrage est associée à l'importance du capital social

\footnotetext{
${ }^{6}$ Pour une présentation détaillée de la typologie, voir Nguyen et al. (2004).
} 


\section{Tableau 1. Déclinaison des différents types de capitaux en indicateurs de pratiques agricoles.}

Table 1. Identification of agricultural practices indicators related to different types of capital.

\section{Capital social}

Réseau des relations - liens forts

- Nombre de personnes $1^{\mathrm{er}}$ cercle de confiance

- \% chiffre d'affaires réalisé en actions collectives locales (transformation ou vente collectives, etc.)

- Nombre de responsabilités dans des structures professionnelles et extraprofessionnelles

Réseau des relations - liens faibles

- Nombre de personnes $2^{\mathrm{e}}$ cercle de confiance

- Nombre d'adhésions à des structures professionnelles et extraprofessionnelles

- Nombre de visites d'exploitations par an

Confiance

- Importance accordée aux relations avec voisins (réponse de l'agriculteur sur une échelle de 1 à 5)

- Importance accordée à l'objectif d'être introduits dans les réseaux (réponse de l'agriculteur sur une échelle de 1 à 5)

\section{Capital naturel}

Indicateurs globaux

- \% chiffre d'affaires réalisé en valorisation non agricole du capital naturel (agrotourisme, bois, etc.)

- Meilleure valorisation des facteurs terre et eau (MBS/ha de SAU et MBS $/ \mathrm{m}^{3}$ d'eau si irrigation)

- Contribution à la pression sur le milieu (spécialisation/diversification du système de production)

- Nombre d'actions environnementales pratiquées

Eau

- N/ha de surface agricole utile (SAU) recevant de l'azote

- Quantité d'eau d'irrigation/ha

Sol

- Nombre d'hectares nus en hiver

Biodiversité

- Nombre d'espèces végétales cultivées

- Nombre d'espèces animales élevées

\section{Capital humain}

- Dimension humaine de l'entreprise (nombre d'UTH, évolution sur les cinq dernières années)

- Importance du savoir-faire spécifique dans le processus de production (réponse agriculteur sur une échelle de 1 à 5)

- Niveau de formation initiale

- Perspectives de succession

- Motivations au métier d'agriculteur

\section{Capital commercial}

- \% chiffre d'affaires de vente directe ou de vente en circuit court

- Contractualisation individuelle avec des clients, générant une plus value (\% chiffre d'affaires)

- Développement d'un réseau de clientèle sur les cinq dernières années

- Utilisation d'intrants locaux

Autres capitaux (physique, financier, etc.)

- Transformation des produits agricoles (\% chiffre d'affaires)

- Location de logements locaux

- Valorisation des bâtiments d'intérêt architectural

UTH : unité de travail humain. 
de l'agriculteur (forte implication dans les réseaux professionnels et extraprofessionnels) et à la présence d'un savoir-faire familial accumulé et valorisé dans les activités. À la différence de la première dynamique, qui est liée à un changement à un moment donné, la seconde dynamique va s'opérer par un processus d'accumulation-valorisation progressive du capital social et du capital humain.

Du point de vue théorique, les hypothèses de la théorie des parties prenantes peuvent éclairer l'analyse des deux dynamiques d'ancrage identifiées. En effet, pour la première dynamique, le choix de valoriser et de préserver les ressources territoriales, en particulier le capital naturel, par des systèmes de production diversifiés n'obéit pas uniquement à des considérations internes à l'exploitation. Elle est aussi en lien avec les attentes et les actions d'influence des parties prenantes de l'exploitation. La question a été posée aux trente agriculteurs déclarant avoir des pratiques de préservation de l'environnenote, sur une échelle de 1 à 5, exprimant l'importance accordée à chaque catégorie de parties prenantes ayant motivé les engagements environnementaux de ces agriculteurs. L'analyse des résultats (tableau 2) fait apparaître deux points importants : le premier, l'action environnementale des agriculteurs n'est pas uniet aux incitations publiques; elle est aussi le fruit de l'influence des parties prenantes agricoles. Le second point, certes les parties prenantes influentes sont $\mathrm{du}$ tionsprofessionnelles agricoles (OPA), clients et fournisseurs), mais d'autres types de parties prenantes commencent à ment. Les agriculteurs ont donné une quement une réponse à la réglementation monde agricole (agriculteurs, organisa-

avoir de l'influence sur l'action des agriculteurs. C'est le cas des acteurs du monde de l'environnement, des voisins non-agriculteurs ou - de moindre importance - les médias

Pour la seconde dynamique d'ancrage, l'accumulation progressive du capital social fait intervenir plusieurs parties prenantes appartenant à différents mondes. On peut citer le monde professionnel agricole via, par exemple, les actions collectives de valorisation des facteurs de production ou des produits, ou le monde extraprofessionnel comme l'ouverture de l'exploitation sur les acteurs voisins non agricoles ou l'implication de l'agriculteur dans les affaires de la cité (associations non agricoles, mairie, etc.).

\section{Conclusion}

L'objectif de cet article était de proposer un nouveau cadre d'analyse de l'exploitation agricole adaptée aux exigences de la durabilité de l'agriculture. Ce cadre s'appuie sur l'approche fondée sur les ressources et sur la théorie des parties prenantes. Ce dernier courant postule que l'exploitation agricole et son fonctionnement ne sont pas simplement une affaire personnelle de l'agriculteur, mais qu'ils concernent aussi bel et bien, à des degrés variables, d'autres acteurs du monde agricole et non agricole. Par conséquent, l'exploitation agricole voit ses frontières classiques modifiées et s'ouvre à de nouveaux acteurs sur le territoire. Si cette vision théorique est en cohérence avec les données empiriques des nouvelles orientations ( $c f$. la modifi-

\section{Tableau 2. Parties prenantes ayant fortement motivé les engagements environnementaux des agriculteurs.}

Table 2. Stakeholders strongly motivating farmers' environmental commitments.

\begin{tabular}{lc}
\hline \multicolumn{1}{c}{ Type de parties prenantes } & \% des réponses \\
\hline Famille et salariés & 9,8 \\
Coop., Groupements de producteurs, clients directs, & 17,6 \\
fournisseurs, banques & \\
Administration (DDAF, ADASEA, Collectivités territoriales) & 23,5 \\
Agriculteurs voisins et organisations professionnelles agricoles & 23,5 \\
Voisins non-agriculteurs & 7,8 \\
Diren, associations environnementales, parcs naturels & 13,7 \\
Médias & 3,9
\end{tabular}

DDAF : direction départementale de l'agriculture et de la forêt; Adasea : association départementale pour l'aménagement des structures des exploitations agricoles; Diren : direction régionale de I'environnement. cation de la composition des CDOA), soulignons toutefois que cette ouverture n'est pas complètement acquise. Ce n'est qu'un processus qui en est à ses débuts, ce qui invite à être prudent et à prendre en compte la dimension temporelle de ce processus qui sera jalonné sans doute de difficultés, de conflits, mais aussi de coopération et d'apprentissage collectif.

L'approche fondée sur les ressources propose un modèle de l'exploitation qui permet à l'agriculteur de trouver dans le nouveau contexte d'agriculture durable des avantages et de les saisir avec une attitude positive en valorisant de nouveaux capitaux, comme le capital naturel et le capital social. Mais là aussi, cette attitude d'agriculteurs n'est pas complètement acquise. Plusieurs obstacles, relevant des normes professionnelles du métier d'agriculteur ou de compétences managériales, sont à surmonter. Ce changement de raisonnement stratégique chez les agriculteurs nécessite une volonté et des capacités d'innovation, ainsi que de nouvelles compétences.

\section{Références}

Aït Abdelmalek A. L'exploitation familiale agricole : entre permanence et évolution. Economie Rurale 2000 ; 255-256 : 40-52.

Arrègle JL. Analyse Resource Based et identification des actifs stratégiques. Revue Française de Gestion 1996 ; 108 : 25-36.

Barney J. Firm resources and sustained competitive advantage. J Manage 1991; 17 : 99-120.

Barthez A. Famille, travail et agriculture. Paris : Economica, 1982

Beletti G, Brunori G, Marescotti A, Rossi A. Individual and collective levels in multifunctional agriculture. Communication au colloque "Systèmes agro-alimentaires localisés: produits, entreprises et dynamiques locales", duits, entreprises et dynamiques
Montpellier, 16-18 octobre 2002 .

Bonneviale JR, Jussiau R, Marshal E. Approche globale de l'exploitation agricole. Dijon: Institut national de recherche et d'applications pédagogiques (Inrap)-Foucher, 1989.

Brossier J, Chia E, Marshall E, Petit M. Gestion de l'exploitation agricole familiale : éléments théoriques et méthodologiques. Dijon : École nationale d'enseignement supérieur agronomique de Dijon (Enesad), Centre national d'études et de ressources en technologies avancées (Cnerta), 2003.

Chia E. Les pratiques de trésorerie des agriculteurs. La gestion en quête d'une théorie. Thèse de doctorat à l'université de Bourgogne, Faculté de science économique et de gestion, Dijon, 1987.

Chombart de Lauwe J, Poitevin J, Tirel JC. Nouvelles gestion des exploitations agricoles. Paris : Dunod, 1964.

Coleman JS. Social capital in the creation of human capital. AJS $1988 ; 94$ : 95-120. 


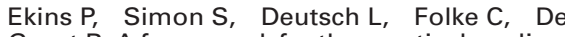
Groot R. A framework for the practical application of the concepts of critical natural capital and strong sustainability. Ecol Econ $2003 ; 44$ 165-85.

Freeman RE. Strategic management : a stakeholder approach. Boston : Pitman, 1984.

Gafsi M. Multifonctionnalité de l'agriculture et redéfinition du rapport de l'exploitation agricole au territoire. In: Barthélemy $D$, Delorme H, Losch B, Moreddiu C, Nieddu M, eds. La multifonctionnalité de l'activité agricole et sa reconnaissance par les politiques publiques. Dijon: Société Française d'Economie Rurale (SFER); Educagri; Centre de coopération internationale en recherche agronomique pour le développement (Cirad), 2003.

Gafsi M, Legagneux B, Nguyen G. Agriculture territorialisée et développement territorial: une analyse exploratoire des transformations du rapport de l'agriculture au territoire en Mu rapport de l'agriculture au territoire en DADP, Montpellier 17-18 décembre 2002.

Gasson R, Errington A. The farm family busi ness. Wallingford: CAB International, 1993.

Godard O, Hubert B. Le développement dura ble et la recherche scientifique à I'INRA. Rapport intermédiaire de mission. Paris : Inra éditions, 2002.

Gray J. Family farms in the Scottish borders : a practical definition by hill sheep farmers. Rural Stud $1998 ; 14: 341-56$
Hagedorn K, Arzt K, Peters U. Institutiona arrangements for environmental co-operatives : a conceptual framework. In : Hagedorn K, ed Environmental Cooperation and Institutional Change. Cheltenham (UK) : Edward Elgar, 2002.

Jonker J, Foster D. Stakeholder excellence? Framing the evolution and complexity of a stakeholder perspective of the firm. Corporate Social Responsability and Environmental Management $2002 ; 9$ : 187-95.

Kay RD, Edwards WM. Farm management Boston : McGraw-Hill, 1999.

Landais E. Agriculture durable: les fondements d'un nouveau contrat social. Courrie de l'environnement de I'INRA 1998 ; 33 : 5-22.

Laurent C, Maxime F, Mazé A, Tichit M. Multi fonctionnalité de l'agriculture et modèles de I'exploitation agricole. Economie Rurale 2003 135-52; (273-274).

Legg W. Sustainable agriculture : an economic perspective Communication au Colloque perspective. Communication au Colloque ADAS "Agriculture and Environment. Challenavril 1999, University of Warwick, UK.

Marshall E, Bonneviale R, Francfort I. Fonc tionnement et diagnostic global de l'exploitation agricole. Une méthode interdisciplinaire pour la formation et le développement. Dijon : École nationale d'enseignement supérieur agronomique de Dijon (Enesad), Service d'édiagronomique de Dijon (Enesad),
tion et de diffusion (SED), 1994.

Mitchell RK, Agle BR, Wood DJ. Toward a theory of stakeholder identification and salience: defining the principle of how and what really counts. Acad Manage Rev 1997 $22: 853-86$.
Nahapiet J, Ghoshal S. Social Capital, Intellectual Capital, and the Organizational Advan tage. Acad Manage Rev 1998 ; 23 : 242-66.

Nguyen G, Gafsi M, Legagneux B. Exploitation agricole et développement territorial : quelles relations les exploitations entretiennent-elles avec leurs territoires ? Communication au colloque SFER, Paris 18-19 novembre 2004.

Organisation de coopération et de développement économiques (OCDE). Multifunctionalité. Paris: OCDE, 2005. http ://www.oecd.org, [ 10 mai 2005 ].

Pretty J. Social capital and the collective management of resources. Science 2003 302 : 1912-4.

Pretty J, Hine R. What is Sustainable Agriculture? In : Pretty J, Hine R, eds. Reducing Food Poverty with Sustainable Agriculture : A Summary of New Evidence. Essex: CES Report. UK : University of Essex, 2002.

Putnam R. Bowling Alone. The Collapse and Revival of American Community. New York : Simon and Schuster, 2000.

Rémy J. La co-institution des contrats territoriaux d'exploitation. Ingénieries, 2001 ( $N^{\circ}$ spécial Multifonctionnalité) ; 45-54.

Russo MV. The emergence of sustainable industries : building on natural capital. Strateg Manage J $2003 ; 24$ : 317-31.

Sharma S. L'organisation durable et ses stakeholders. Revue Française de Gestion 2001 136 : 154-67.

Wernerfelt B. A resource-based view of the firm. Strateg Manage J 1984 ; 5 : 171-80.

Wernerfelt B. The resources-based view on the firm: ten years after. Strateg Manage $J$ $1995 ; 16: 171-4$. 